INEL-95/0025

\title{
Limitations for Heterodyne Detection of Brillouin Scattered Light
}

\author{
R. T. Allemeier \\ J. W. Wagner \\ K. L. Telschow
}

Published January 1995

Idaho National Engineering Laboratory Lockheed Idaho Technologies Company Idaho Falls, Idaho $\mathbf{8 3 4 1 5}$ 


\begin{abstract}
Heterodyne methods for detection of Brillouin-scattered light are considered one possible means to increase the speed of the scattered light frequency detection. Results of experiments with simulated Brillouin scattering suggest that heterodyne detection of the Brillouin-scattered light is feasible. Experiments to detect Brillouin-scattered light, with water as the scattering medium, were designed and interpreted using the results of the simulated scattering experiments. Overall, results showed that it is difficult to narrow the linewidth for Brillouin scattering to an acceptable level. The results given indicate that heterodyne detection of the Brillouin components requires detection bandwidths that are quite small, perhaps $10 \mathrm{~Hz}$ or lower. These small bandwidths can be routinely achieved using lock-in amplifier techniques.
\end{abstract}

\title{
DISCLAIMER
}

This report was prepared as an account of work sponsored by an agency of the United States Government. Neither the United States Government nor any agency thereof, nor any of their employees, makes any warranty, express or implied, or assumes any legal liability or responsibility for the accuracy, completeness, or usefulness of any information, apparatus, product, or process disclosed, or represents that its use would not infringe privately owned rights. Reference herein to any specific commercial product, process, or service by trade name, trademark, manufacturer, or otherwise does not necessarily constitute or imply its endorsement, recommendation, or favoring by the United States Government or any agency thereof. The views and opinions of authors expressed herein do not necessarily state or reflect those of the United States Government or any agency thereof. 


\section{DISCLAIMER}

Portions of this document may be illegible in electronic image products. Images are produced from the best available original document. 


\section{CONTENTS}

ABSTRACT $\ldots \ldots \ldots \ldots \ldots \ldots \ldots \ldots \ldots \ldots \ldots \ldots \ldots \ldots \ldots \ldots \ldots \ldots$

INTRODUCTION $\ldots \ldots \ldots \ldots \ldots \ldots \ldots \ldots \ldots \ldots \ldots \ldots \ldots \ldots \ldots \ldots$

IMPORTANT PROPERTIES OF BRILLOUIN-SCATTERED LIGHT $\ldots \ldots \ldots \ldots \ldots \ldots$

EXPERIMENTAL DESIGN AND RESULTS $\ldots \ldots \ldots \ldots \ldots \ldots \ldots \ldots \ldots$

CONCLUSIONS $\ldots \ldots \ldots \ldots \ldots \ldots \ldots \ldots \ldots \ldots \ldots \ldots \ldots \ldots \ldots \ldots \ldots \ldots$

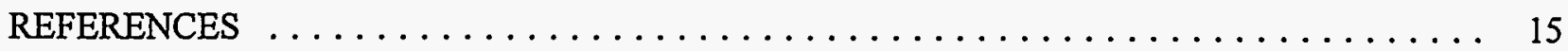

\section{FIGURES}

1. Geometric representation of the Anti-Stokes and Stokes components of Brillouin scattering

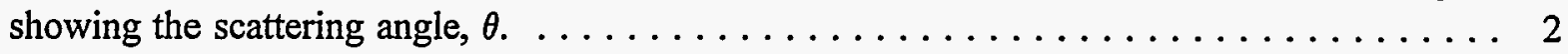

2. The optical setup used for the simulated Brillouin-scattering experirnents. $\ldots \ldots \ldots \ldots$

3. The scattering geometry for the simulated Brillouin-scattering experiments. . . . . . . 7

4. A series of Doppler shifts for disk rotation rates of $15,10,5$, and $0 \mathrm{~Hz} \ldots \ldots \ldots$

5. The optical setup used for the Fabry-Perot detection of the Brillouin components. . . . . 10

6. The scattering cell used for the Brillouin-scattering experiments. $\ldots \ldots \ldots \ldots \ldots$

7. The fiberoptic based heterodyne detection system. $\ldots \ldots \ldots \ldots \ldots \ldots \ldots$

8. The amplitude of the beat frequency for a scan of the piezoelectric transducer in $100 \mathrm{kHz}$

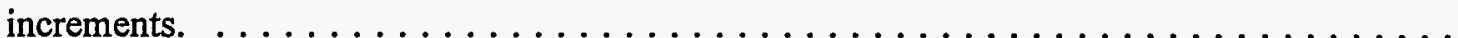

\section{TABLES}

1. Minimum detectable signal beam powers using heterodyne detection for different linewidths . 8 


\section{Limitations for Heterodyne Detection of Brillouin Scattered Light}

\section{INTRODUCTION}

One means by which elastic properties of a material may be determined is measuring sound wave velocities in the material, from which elastic moduli of interest can be computed. Velocity can be measured by conventional piezoelectric transduction techniques, by applying laser ultrasonics, or by using Brillouin-scattering methods ${ }^{1-7}$.

Brillouin scattering of light arises from the interaction of photons with the thermal phonons present in the material ${ }^{8}$. This photon-phonon interaction alters the frequency imparted to the scattered light; the frequency shift is angularly dependent as can be seen from the following well known relationship ${ }^{8}$.

$$
\Delta \omega= \pm \frac{2 n v_{s} \omega_{o}}{C_{o}} \sin \frac{\theta}{2}
$$

where

$\mathrm{n}=$ the index of refraction of the medium

$v_{s}=$ the sound wave velocity in the material

$\omega_{0}=$ the angular frequency of the light

$C_{o}=$ the speed of light in vacuum

$\theta=$ the scattering angle as shown in Figure 1.

This relation shows that the scattered light consists of a doublet with the magnitude of the frequency shift given by the frequency of the scattering sonic wave. In practice, the quantities $n$, $C_{o}$, and $\omega_{o}$ are known prior to making Brillouin-scattering measurements, while the quantities $\Delta \omega$ and $\theta$ are measured directly, allowing the sound wave velocity in the material to be calculated.

Brillouin-scattering techniques for determining the sound wave velocity are particularly attractive since they are completely noninvasive. Only a probe beam of light is required since the thermal energy in the material provides the elastic motion. Therefore, very little energy is added to the material and no contact is made with it. In contrast, laser ultrasonic icsieidiques and piezoelectric detection methods deliberately add acoustic energy to the material. The ability to focus the Brillouin probe beam implies that the technique could be used to probe single grains in a sample where the grains are dimensionally on the order of the focused probe beam spot size. The technique could be used as a "microscope" based upon local sound wave velocity and thus on local elastic properties.

It would also be advantageous to use the Brillouin-scattering technique in process control applications. For example, one could monitor the properties of the resin in composite materials 

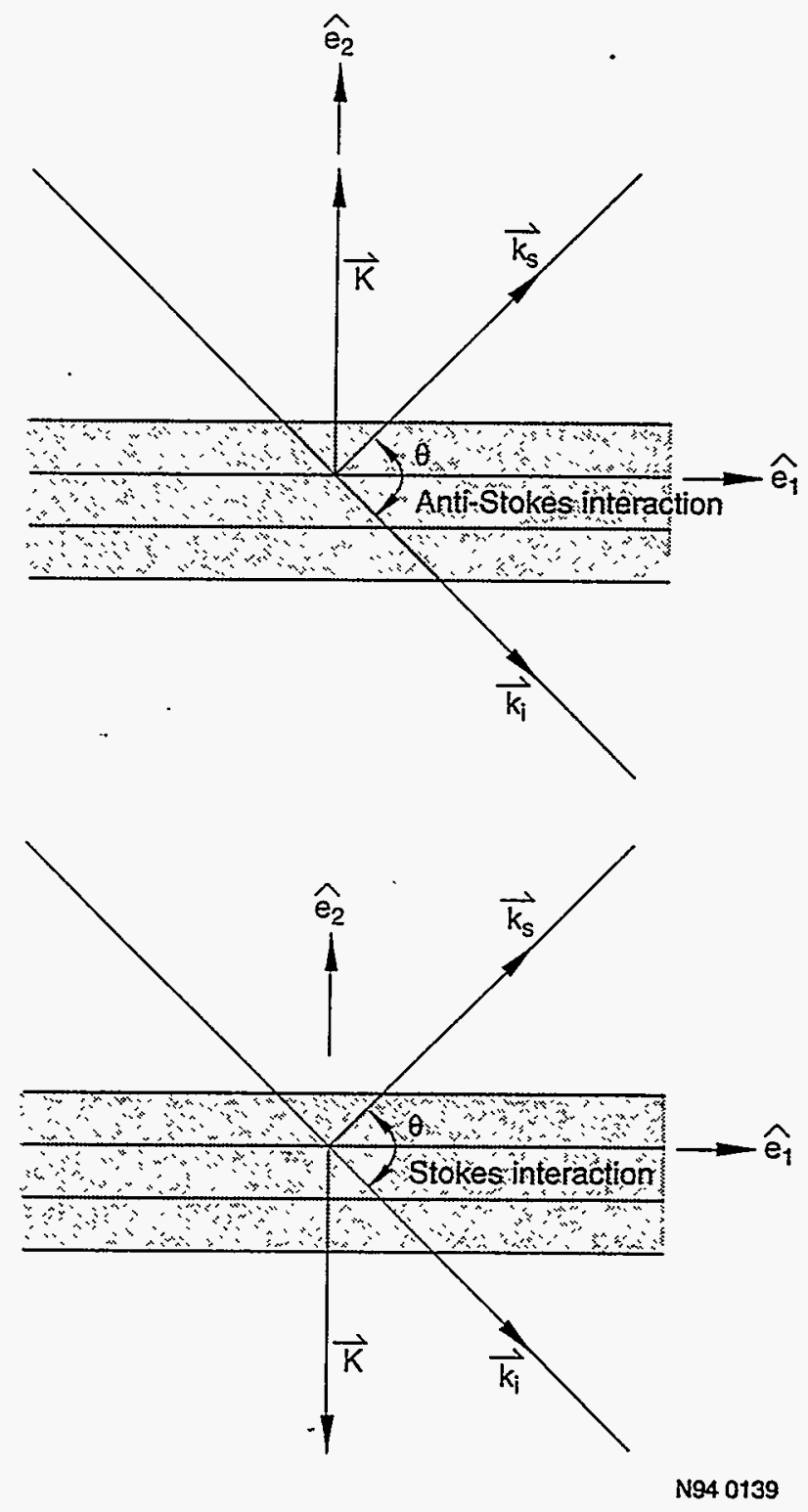

Figure 1. Geometric representation of the Anti-Stokes and Strkes components of Brillouin scattering showing the scattering angle, $\theta$. 
during curing to provide feedback for autoclave temperature and pressure control. To achieve this real-time monitoring, the detection needs to be fast, having measurement periods in the neighborhood of 1 or 2 min. Heterodyne detection is an excellent candidate for this. It has the advantage of being nearly 2 orders of magnitude faster than video detection techniques ${ }^{9}$, and is also able to measure the linewidth of the scatterer. The linewidth, in turn, gives the attenuation of sound at a particular frequency ${ }^{10,11}$.

This paper discusses factors that must be considered when attempting heterodyne detection of Brillouin-scattered light. 


\section{IMPORTANT PROPERTIES OF BRILLOUIN-SCATTERED LIGHT}

The amplitude of the scattered electric field for Brillouin scattering is ${ }^{12}$

$$
E_{s}(\vec{R}, t)=\frac{k_{s}^{2}}{4 \pi R}\left(\frac{\epsilon_{o}}{\epsilon}\right) \sin \phi E_{i} e^{i\left(\omega_{i} t-\vec{k}_{s} \cdot \vec{R}\right)} \int_{\nu} \Delta \chi\left(\vec{r}_{o}, t\right) e^{i\left(\vec{k}_{s}-\vec{k}_{i}\right) \vec{r}^{o}} d^{3} \vec{r}_{o}
$$

where,

$\mathrm{k}_{\mathrm{s}} \quad=$ the magnitude of the scattered wavevector

$\mathrm{R}=$ the distance from the scattering center to the point of observation

$\phi=$ the angle between the incident electric field vector and the plane of observation

$\omega=$ the angular frequency of the incident electric field

$\Delta \chi=$ the fluctuation in susceptibility as a function of the phonon wavevector and time.

The susceptibility fluctuations create the time varying inhomogeneity required to produce the scatter. These fluctuations decay exponentially, therefore the lineshape of the scattered electric field is Lorentzian ${ }^{11}$ with a half width equal to the inverse of the decay time. The effect of this lineshape is to alter the temporal coherence properties of the scattered beam relative to those of the incident beam. In addition to the change in temporal coherence, the spatial coherence of the scattered beam is altered owing to the short correlation length ${ }^{10}$ of the susceptibility fluctuations. Therefore, one property of the scattered light is that the coherence of the scattered beam has been altered by the scattering process.

The second property of the scattered light that is of importance is the scattering efficiency (ratio of the scattered power to the incident optical power). This efficiency is, of course, material dependent. In general, the scattering efficiency for liquids can be taken to be approximately $10^{-12}$ for a particular scattering angle ${ }^{8}$. This indicates that, for a given scattering angle, a $1 \mathrm{~W}$ incident beam will produce a $1 \mathrm{pW}$ scattered beam.

The challenge in heterodyne detection of Brillouin-scattered light is to provide adequate sensitivity given the coherence and power limitations described above. To quantify the difficulties in making a measurement of this sort, three sets of experiments were designed; these will be discussed below. 


\section{EXPERIMENTAL DESIGN AND RESULTS}

For the experiments to be discussed below, the objective was to heterodyne detect the Brillouin components scattered from nearly pure water. The scattering angle chosen for this detection was nominally $0.75^{\circ}$ which, according to Equation 1, should yield a frequency shift of $\pm 50 \mathrm{MHz}$ and a linewidth in the neighborhood of $50 \mathrm{kHz}^{10}$ when the incident probe beam has wavelength of $514.5 \mathrm{~nm}$. Therefore, the experiments were designed to evaluate the detection capability under these conditions.

As mentioned, there were three sets of experiments that were designed to evaluate the difficulties in heterodyne detection of Brillouin-scattered light. The first of these experiments was a simulated Brillouin scattering. This was accomplished by scattering light from a rotating diffuse surface. In this case, both the time varying spatial coherence properties and the amount of scattered power could be precisely controlled. By collecting light scattered from an relatively large area on the surface (approximately $2 \mathrm{~mm}$ in diameter), the spatial coherence properties of the scattered light were controlled. The experimental setup for this is shown in Figure 2. This arrangement allowed for a difference frequency of nominally $5 \mathrm{MHz}$ to be maintained between the local oscillator and probe arms of the interferometer via the two acousto-optic modulators (AOM). In addition, the AOM in the probe arm of the interferometer allowed the light scattered from the moving surface to be precisely controlled, by controlling the AOM drive voltage.

Figure 3 shows the geometry for scattering from the moving surface. The Doppler shift produced by scattering the incident beam from a surface in motion is given by

$$
v_{D}=\frac{1}{2 \pi}\left(\vec{k}_{i}-\vec{k}_{s}\right) \vec{v}
$$

Using the geometry shown in Figure 3, Equation 3 reduces to

$$
v_{D}=\frac{r \omega}{\lambda} \sin \beta \sin \theta_{s}
$$

The broadening of the spectrum of the scattered light can be computed from Equation 4 by calculating the various differentials as given in Equation 5.

$$
\Delta v= \pm\left[\left(\frac{\Delta v_{c}}{2}\right)^{2}+\left(\frac{\partial v_{D}}{\partial r} \Delta\right)^{2}=\left(\frac{\partial v_{D}}{\partial \beta} \Delta \beta\right)^{2}\right]^{\frac{1}{2}}
$$

where $\Delta v_{C}$ is the broadening produced by the correlation time of the surface, which is given as $r \omega / s^{13}$, where $s$ is the dianieter of the scattering area. The effect of the correlation time is small compared to the broadening produced by the range of velocities in the scattered beam resulting from the extended collection area and the particular scattering geometry used.

The assumption has been made that the incident beam is well collimated and normal to the scattering surface. It is also assumed that the scattered beam and the incident beam are both in the horizontal plane. Figure 4 shows the spectra of four different beat frequencies obtained from heterodyne detection of scattered light from the diffuse surface when it is rotating at $15,10,5$, and $0 \mathrm{~Hz}$. These data show the expected increase in Doppler shift and linewidth with increasing 


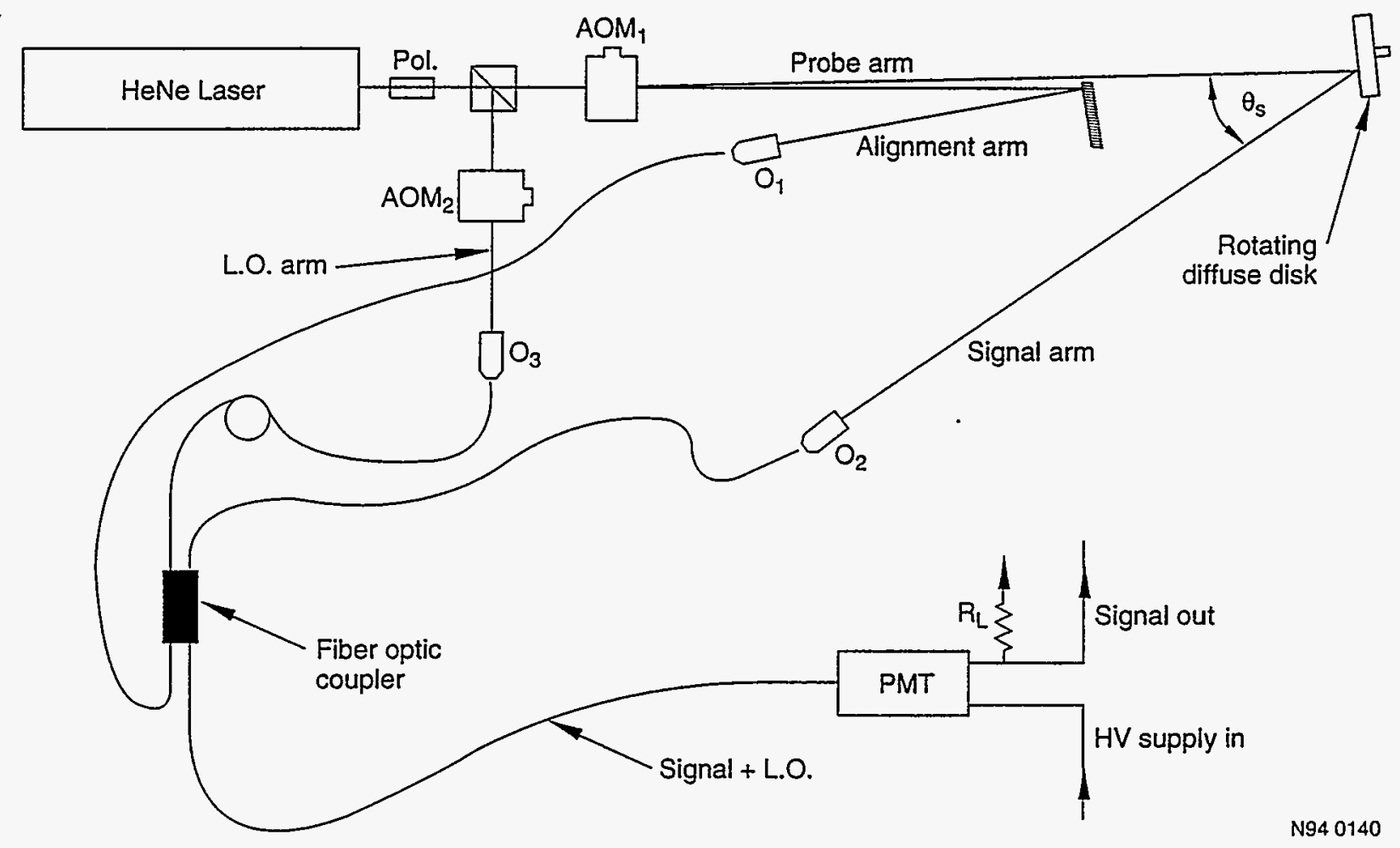

Figure 2. The optical setup used for the simulated Brillouin-scattering experiments. $A O M_{n}$, acousto-optic modulator; $O_{n}, 20 x$ microscope objectives; Pol., Glan-Thompson polarizer. 

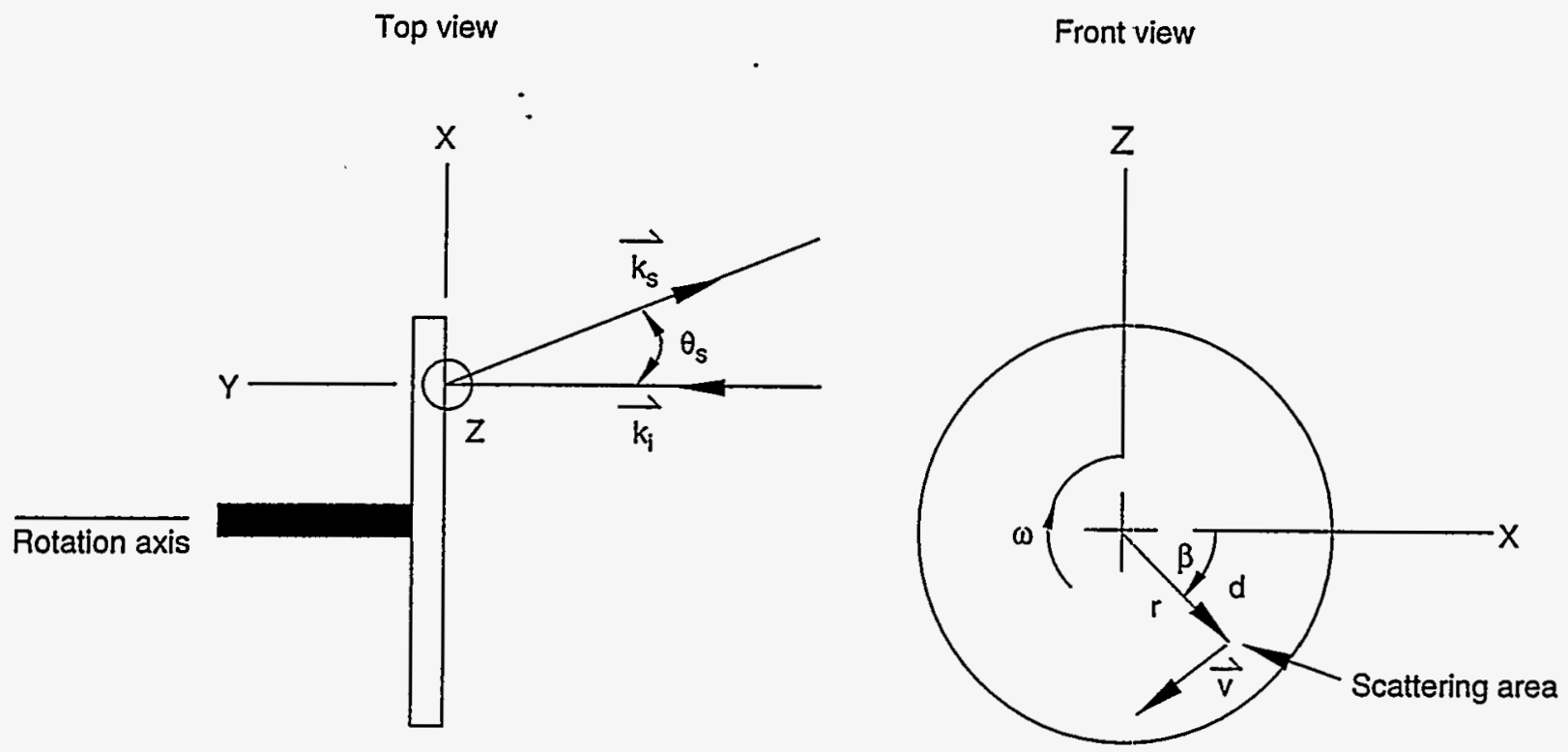

N94 0141

Figure 3. The scattering geometry for the simulated Brillouin-scattering experiments, showing the location of the scattering point relative to the rotation axis of the disk.

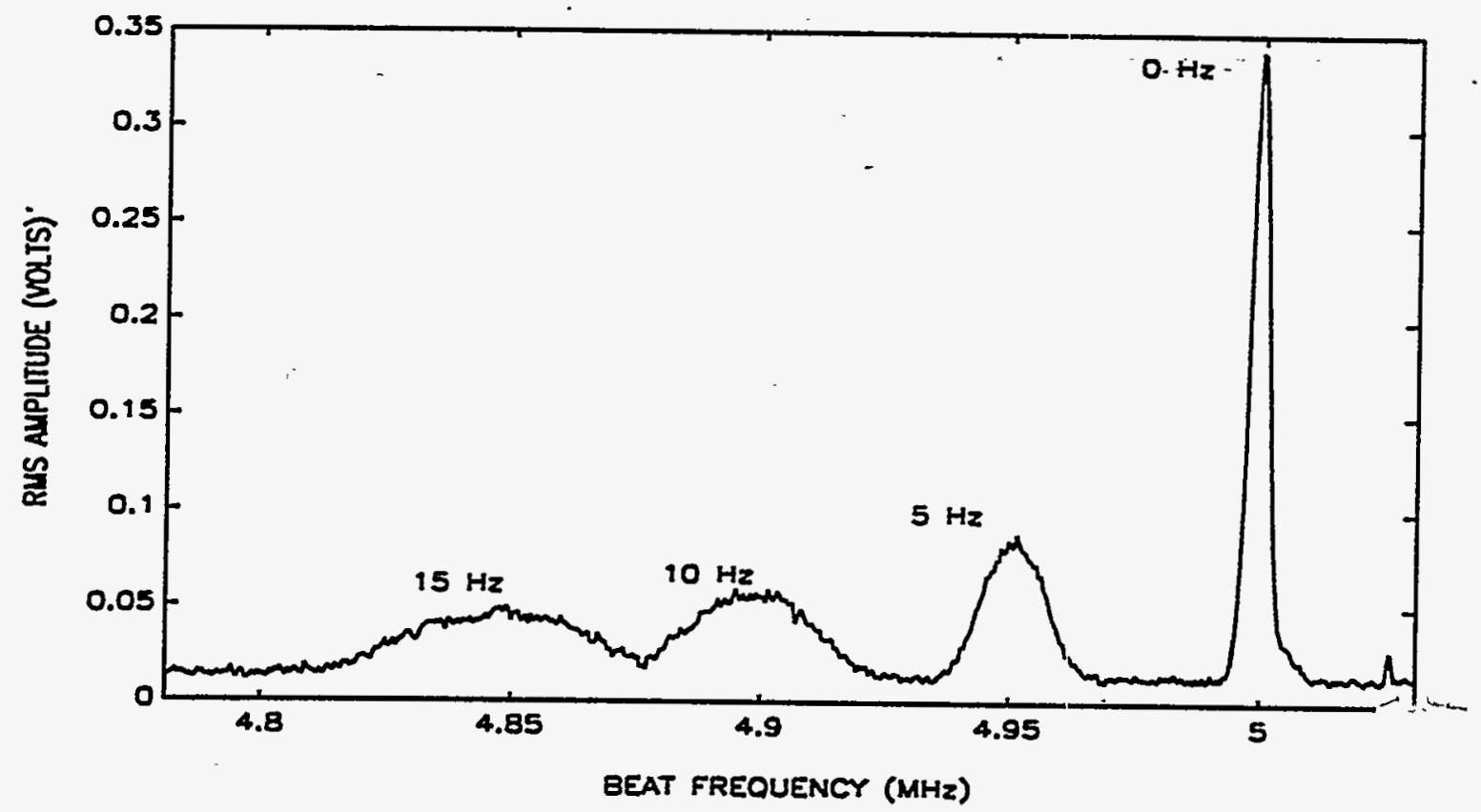

Figure 4. A series of Doppler shifts for disk rotation rates of $15,10,5$, and $0 \mathrm{~Hz}$. The peak at the far right in the plot is for the $0 \mathrm{~Hz}$ rotation rate. of the disk; therefore, the Doppler shift is also zero, leaving the beat frequency unchanged. The peak at the far left is for the $15 \mathrm{~Hz}$ rotation rate and, therefore, the Doppler shift if proportionately higher, resulting a lower beat frequency. 
surface velocity. The reason for a lower beat frequency at higher rotation rates is that the scattered beam suffers an increase in frequency from the moving surface; therefore, the difference frequency between the signal and local oscillator beams is reduced.

Using the scattering arrangement discussed above, minimum detectable power levels for various scattered linewidths were obtained; these are summarized in Table 1 . The detection bandwidth for the spectrum analyzer was chosen to be $3 \mathrm{kHz}$ for all the heterodyne detection experiments. The laser used for the simulated scattering measurements was a Laboratory For Science model 200 frequency-stabilized HeNe laser. Power levels in the signal beam were measured using a Hamamatsu R1477 photomultiplier tube that had been calibrated for both 632.8 and $514.5 \mathrm{~nm}$ light. As can be seen from the data of Table 1, the minimum detectable powers for the expected linewidth that can be detected via heterodyne detection are well below the $1 \mathrm{pW}$ level stated above for liquids.

The significance of the above experiment is the prediction that heterodyne detection of the Brillouin-scattered light should be possible providing the scattered power and linewidth are near those given in Table 1. Subsequent experiments were conducted using the $514.5 \mathrm{~nm}$ line of an argon laser rather than a HeNe as was used for the simulated scattering. experiments. This change was made primarily so that larger probe beam powers could be attained for the actual Brillouin-scattering experiments. The results of the simulated scattering experiments then become a worst case since the quantum efficiency of the R1477 PMT is higher at $514.5 \mathrm{~nm}$ than at $632.8 \mathrm{~nm}$.

Table 1. Minimum detectable signal beam powers using heterodyne detection for different linewidths.

\begin{tabular}{ccccc}
\hline $\begin{array}{c}\text { Disk rotation } \\
\text { rate }(\mathrm{Hz})\end{array}$ & $\begin{array}{c}\text { Surface speed of } \\
\text { disk }(\mathrm{m} / \mathrm{s})\end{array}$ & $\begin{array}{c}\text { Linewidth }(\mathrm{kHz}) \\
0.0\end{array} \mathrm{c}_{-}$ & $\begin{array}{l}\text { Signal beam } \\
\text { power }(\mathrm{W})\end{array}$ & S/N power ratio \\
\hline 0.0 & $0.4 \pm 0.4$ & $3.6 \times 10^{-14}$ & 3.1 \\
$4.0 \pm 0.1$ & $0.19 \pm 0.01$ & $6.6 \pm 0.7$ & $4.7 \times 10^{-14}$ & 2.0 \\
$6.0 \pm 0.1$ & $0.38 \pm 0.02$ & $10.7 \pm 1.1$ & $2.4 \times 10^{-14}$ & 2.0 \\
$8.0 \pm 0.1$ & $0.75 \pm 0.03$ & $21.7 \pm 2.2$ & $26.1 \times 10^{-14}$ & 1.9 \\
$10.0 \pm 0.1$ & $0.94 \pm 0.03$ & $26.9 \pm 2.7$ & $26.0 \times 10^{-14}$ & 2.2 \\
$12.0 \pm 0.1$ & $1.13 \pm 0.04$ & $33.2 \pm 3.3$ & $38.5 \times 10^{-14}$ & 2.0 \\
$14.0 \pm 0.1$ & $1.32 \pm 0.04$ & $40.2 \pm 4.0$ & $33.8 \times 10^{-14}$ & 2.1 \\
$16.0 \pm 0.1$ & $1.51 \pm 0.05$ & $47.0 \pm 4.7$ & $47.4 \times 10^{-14}$ & 1.8 \\
$18.0 \pm 0.1$ & $1.70 \pm 0.06$ & $54.1 \pm 5.4$ & $44.4 \times 10^{-14}$ & 1.9 \\
\hline
\end{tabular}


The second set of experiments was designed to measure the power in an actual Brillouinscattered beam. Bulk optics, as shown in Figure 5, produced the best measurement results for the Fabry-Perot detection method. Independent measurements ${ }^{12}$ indicate that for the fiber coupler used in the simulated scattering experiments the amount of light reacling the detector from the signal beam is approximately $35 \%$ of that incident on the objective. Therefore, it is assumed that when the coupler is used the signal power reaching the detector is $1 / 3$ of the total signal beam power. The primary reason for using fiber optics in these experiments was to aid in maintaining a light-tight enclosure around the photomultiplier tube. A fiber optic coupler also diminishes the difficulties in obtaining proper phase-front matching when combining the local oscillator and signal beams for heterodyne detection.

An enlarged view of the scattering cell is shown in Figure 6. The separation between input and output windows is approximately $70 \mathrm{~cm}$. A $50 \mathrm{MHz}$ piezoelectric transducer with a focal length of $32 \mathrm{~mm}$ was built into the cell to aid in alignment. Since the Brillouin effect is the scattering of light from thermally-generated sound waves of the material, the driven sound waves produced by the transducer will be of the same frequency and scattering angle. Therefore, the transducer makes it possible to align the system with a strong, visible scattered beam.

Using the above described arrangement to measure the scattered power in the Brillouin components and correcting for the $80 \%$ to $90 \%$ loss associated with the Fabry-Perot transmission, the combined measured power in the Stokes and Anti-Stokes components was between $2.5 \mathrm{pW}$ and $5 \mathrm{pW}$ for a $500 \mathrm{~mW}$ probe beam (this probe beam power was maintained for all scattering experiments). These values were obtained with the Stokes and Anti-Stokes components overlapping to improve the signal-to-noise ratio and are in agreement with expected values ${ }^{10}$. Although the power level could be measured, the finesse of the Fabry-Perot was not sufficient to give any insight into the linewidth of the Brillouin components. The measured power levels indicate that when using the fiberoptic coupler in the heterodyne interferometer, the resulting signal beam power level will be between $440 \mathrm{fW}$ and $875 \mathrm{fW}$. These values are based on the facts that the power level measured using the Fabry-Perot was obtained when the Stokes and Anti-Stokes components of the Brillouin-scattered light were overlapping and that only one of the two components will be detected via heterodyne detection. In addition to the above reduction factor of $50 \%$, an additional reduction to $35 \%$ of this value results from the fiberoptic coupler transmittance. Using the data of Table 1, the above stated power levels indicate that if the linewidth is as expected, the signal-to-noise ratio should be mininally 1.7 .

Shown in Figure 7 is the schematic representation of the optical setup used for the third set of experiments. The detection electronics were as shown in Figure 4. This set of experiments was the effort to heterodyne detect the Brillouin-scattered light. In spite of the above predictions, it was not possible to heterodyne detect the Brillouin components.

It was suspected that the lack of detectability was the result of the linewidth being greater than expected. To evaluate this hypothesis, the frequency of the alignment transducer in the cell was scanned. Typical results for this scanning are shown in Figure 8 . They indicate that the actual linewidth of the signal beam was at least $2 \mathrm{MHz}$ rather than $50 \mathrm{kHz}$, an increase by a factor of 40. This increase is a direct result of having a solid angle of collection that is too large. A smaller solid angle of collection is extremely difficult to achieve, as subsequent efforts indicated. 


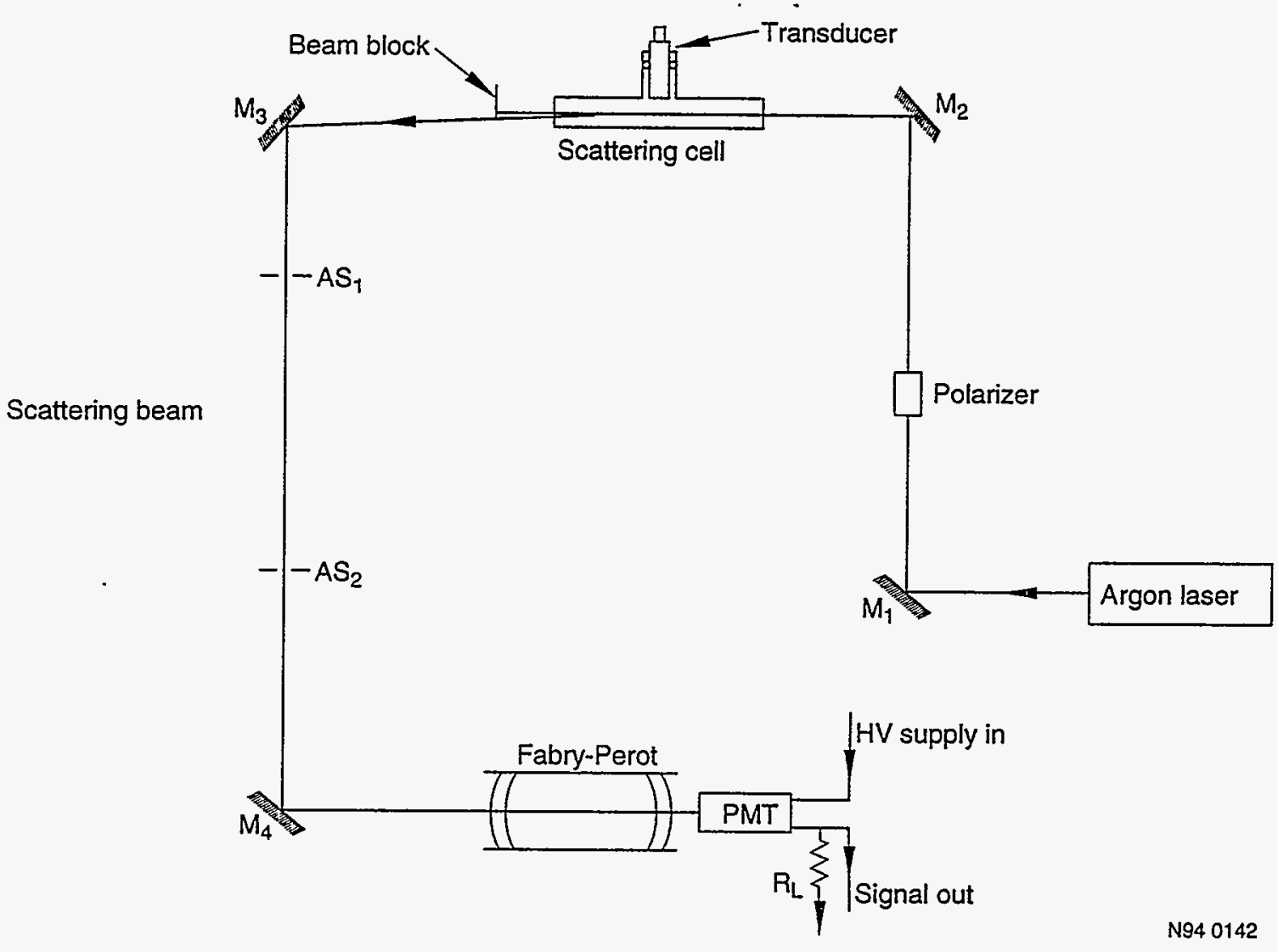

Figure 5. The optical setup used for the Fabry-Perot detection of the Brillouin components. $M_{n}$, aluminized mirrors; $A S_{n}$, adjustable aperture stops. 


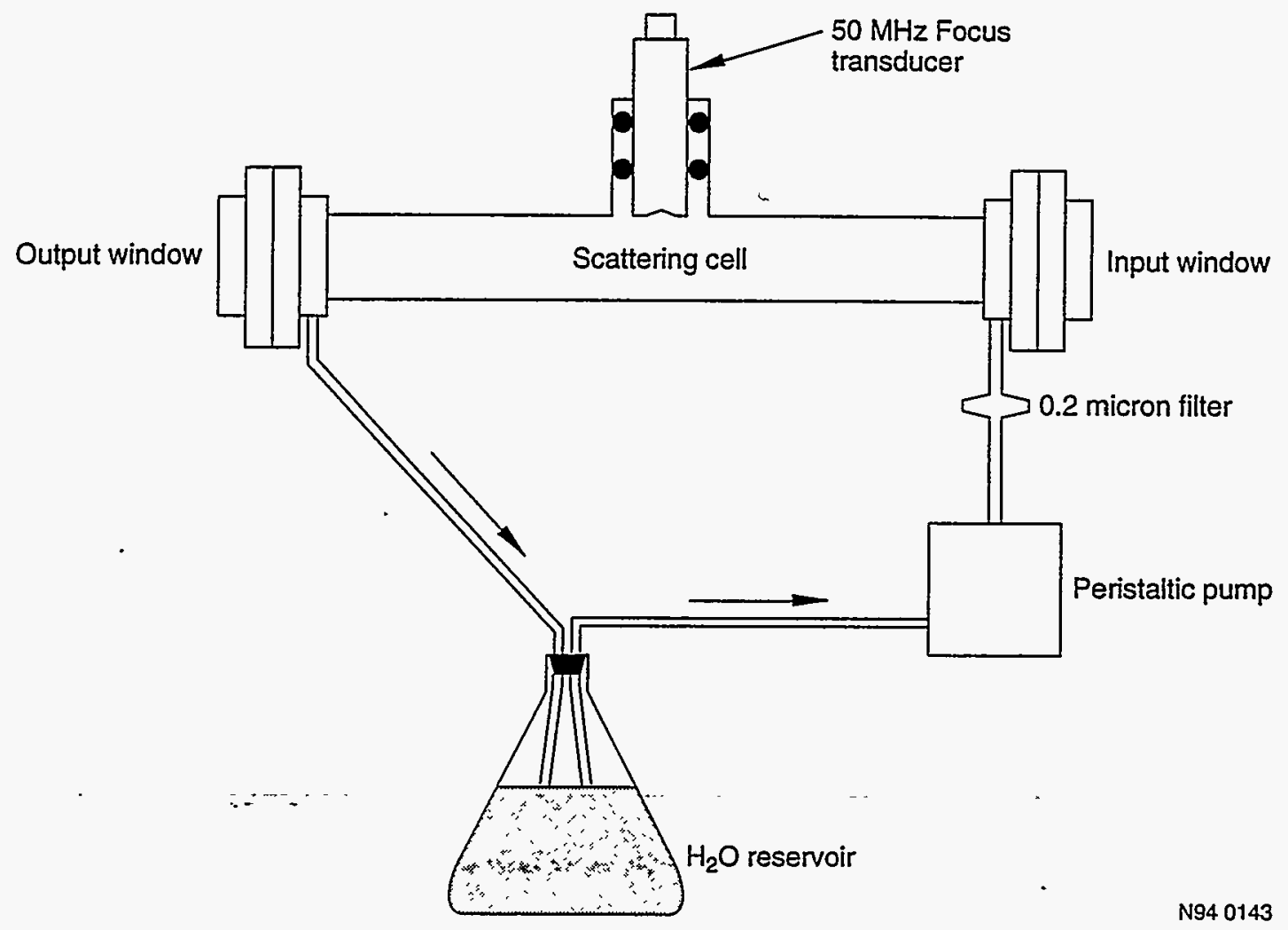

Figure 6. The scattering cell used for the Brillouin-scattering experiments. Continuous filtering of the water was possible with this system, helping to reduce unwanted elastic scatter. The cell was also fitted with a piezoelectric transducer that aided in optical alignments. The pump was turned off during periods of measurement. 


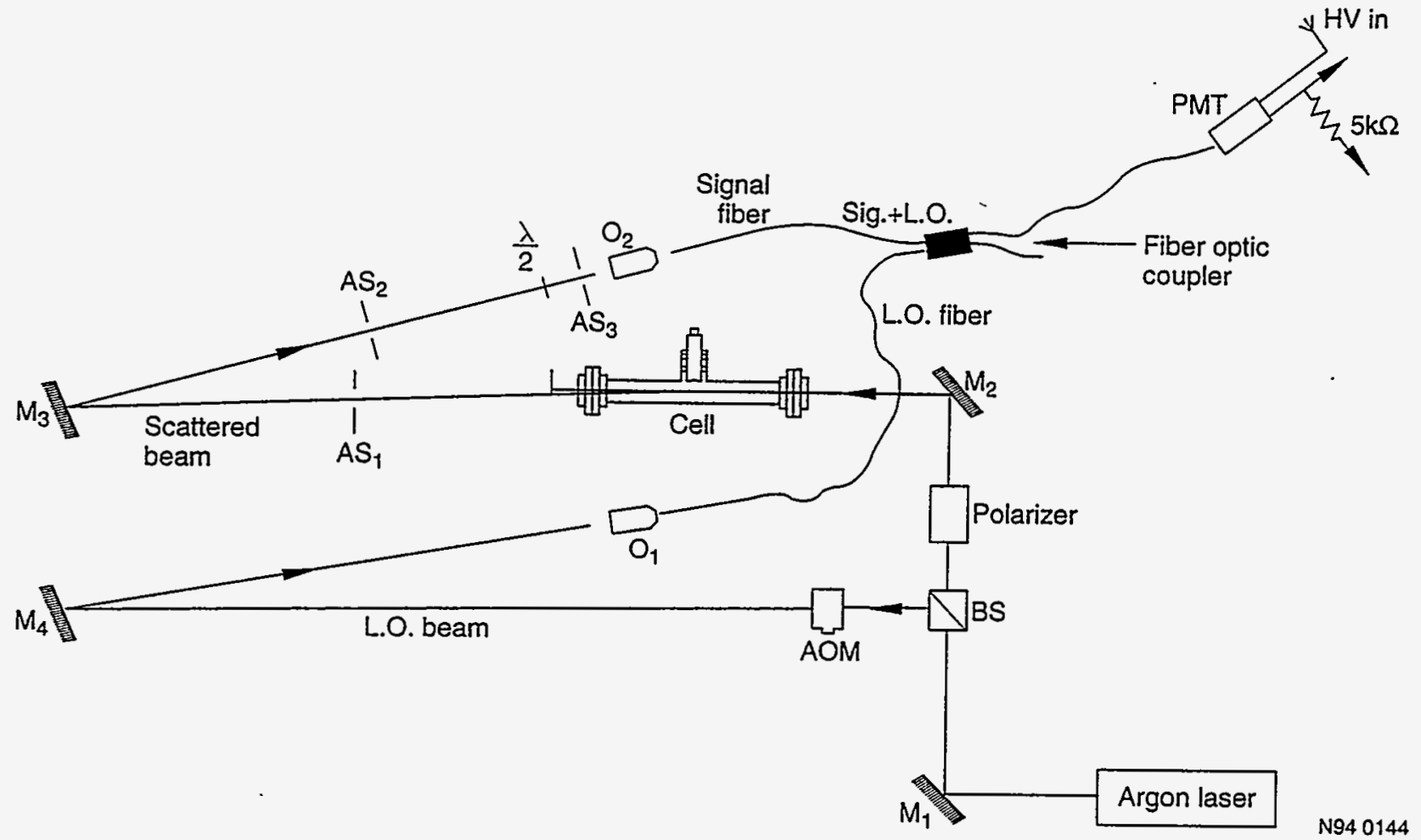

Figure 7. The fiberoptic based heterodyne detection system. $M_{n}$, aluminized mirrors; $B S$, broadband beamsplitter; $A O M$, acousto-optic modulator; $\lambda / 2$, half waveplate; $O_{n}$, microscope objectives.

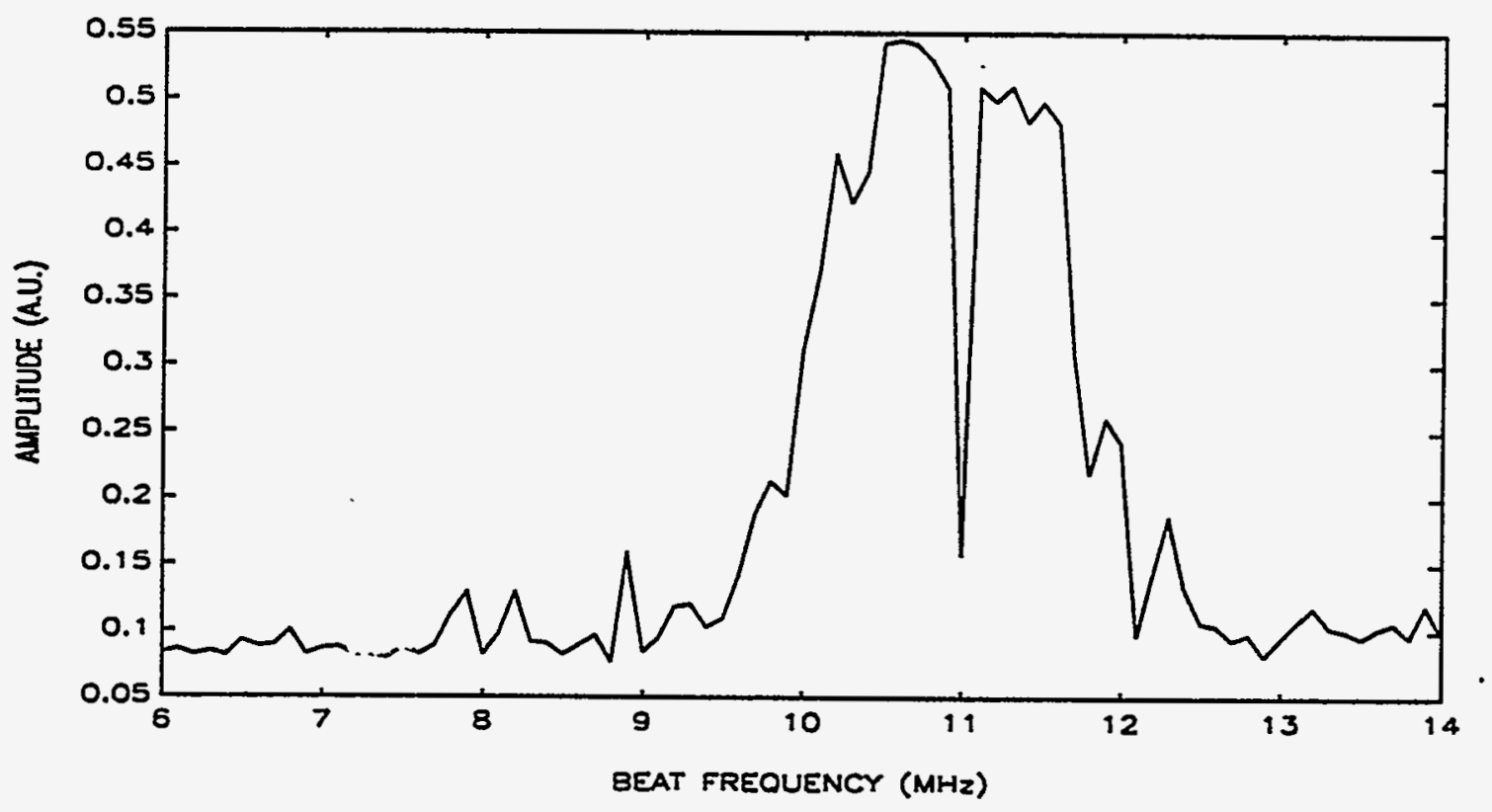

Figure 8. The amplitude of the beat frequency for a scan of the piezoelectric transducer in 100 $\mathrm{kzHz}$ increments. A $20 \mathrm{x}$ microscope objective was used to collect the diffracted beam and inject it into the fiber. 
The results indicate that the $3 \mathrm{kHz}$ detection bandwidth used will be too broad. However, when using a spectrum analyzer the frequency window width and the detection bandwidth are not independent and, therefore, for narrower detection bandwidths the scan times must be slower and the frequency window must be narrower. 


\section{CONCLUSIONS}

Results of the simulated Brillouin-scattering experiments indicate that, in principle, heterodyne detection of Brillouin-scattered light is possible. However, it is difficult, if not impossible, to narrow the linewidth for Brillouin scattering to a level sufficient to provide an adequate signal-to-noise ratio. The results obtained in this study indicate that detection of the Brillouin components via heterodyne methods requires detection bandwidths of $10 \mathrm{~Hz}$ or lower. These small bandwidths can be achieved using lock-in amplifier techniques, with the drawback of a more cumbersome electronic configuration. 


\section{REFERENCES}

1. V. Askarpour and M. H. Manghnani, "Elastic Properties of Diopside, Anorthite, and Grossular Glasses and Liquids: Abrillouin Scattering Study up to $1400 \mathrm{~K}$ ", J. Geophy. Res., vol. 98, no. B10, 17,683-17,689, October 10, 1993.

2. S. S. Bhagat, J. D. Bass, and J. R. Smyth, "Single-Crystal Elastic Properties of OmphaciteC2/c by Brillouin Spectroscopy", J. Geophy. Res., vol. 97, no. B5, 6843-6848, May 10, 1992.

3. J. Xu, R. Wang, M. H. Manghnani, A. Misra, Y. Song, and J. Fi. Gaines, "Ultrasonic Measurements on Silver $(\mathrm{Ag})$-High $\mathrm{T}_{\mathrm{c}}$ Superconductor $\left(\mathrm{YBa}_{2} \mathrm{Cu}_{3} \mathrm{O}_{7-8}\right)$ Composites at pressures up to $0.4 \mathrm{GPa}^{\prime \prime}$, J. Appl. Phys. vol. 70, no. 11, 7182-7184, December 1, 1991.

4. J. D. Bass, "Elasticity of Grossular and Spessartite Garnets by Brillouin Spectroscopy", J. Geophy. Res., vol. 94, no. B6, 7621-7628, June 10, 1989.

5. J.-D. Aussel and J.-P. Monchalin, "Precision Laser-Ultrasonic Velocity Measurement and Elastic Constant Determination", Ultrasonics, vol. 27, no. 3, 166-177, May 3, 1989.

6. M. Cankurtaran, G. A. Saunders, J. R. Willis, A. Al-kheffaji, D. P. Almond, "Bulk Modulus and its Pressure Derivative of $\mathrm{YBaCu}_{3} \mathrm{O}_{7-\mathrm{x}}$ ", Phy. Rev. B, vol. 39, no. 4, 2872-2875, February 1, 1989.

7. A. Polian and M. Grimsditch, "Elastic Properties and Density of Helium up to $20 \mathrm{GPa",}$ Europhys. Lett. vol. 2, no. 11, 849- 55, December 1, 1986.

8. B. Chu, "Laser Light Scattering", Academic Press, 1974, Chap. 3.

9. A. Yariv, "Optical Electronics", $3^{\text {rd }}$ Ed., Holt, Rhinehart, and Winston, Inc., 1985, Chap. 12.

10. G. Benedek and T. Greytak, "Brillouin Scattering in Liquids", IEEE Proc. vol. 53, no. 10, October 1965.

11. J. B. Lastovka, Doctoral Dissertation, Physics, Massachusetts Institute of Technology, 1967.

12. R. T. Allemeier, Doctoral Dissertation, Materials Science and Engineering, Johns Hopkins University, 1993.

13. M. C. 'liech, "Homodyne Detection of Infrared Radiation from a Moving Diffuse Target", IEEE Proc. vol. 57, no. 5, May 1969. 